\title{
Differential gene expression and the importance of regulatory ncRNAs in acidophilic
}

\section{microorganisms}

Daniela S. Aliaga Goltsman ${ }^{1,3 \#}$, Loren Hauser $^{2,4}$, Mauna Dasari ${ }^{1,5}$, Brian C. Thomas ${ }^{1,6}$ and Jillian

\author{
F. Banfield ${ }^{1 \#}$ \\ ${ }^{1}$ University of California Berkeley, Berkeley CA \\ ${ }^{2}$ Oak Ridge National Laboratory, Oak Ridge TN \\ ${ }^{3}$ Current address: Stanford University School of Medicine, Stanford CA \\ ${ }^{4}$ Current address: Digital Infuzion, Inc., Gaithersburg, MD \\ ${ }^{5}$ Current address: University of Notre Dame, Notre Dame, IN \\ ${ }^{6}$ Current address: Metagenomi, Berkeley CA
}

\#Corresponding authors:

Daniela S. Aliaga Goltsman

Jillian Banfield

Stanford University

University of California Berkeley

School of Medicine

Earth and Planetary Sciences Department

Department of Infectious Diseases

Energy Biosciences Building

3801 Miranda Ave., Bldg. 101, Rm B4-185

2151 Berkeley Way

Palo Alto, CA 94304

Berkeley, CA 94720

dgolts@stanford.edu

jbanfield@,berkeley.edu

Submitted as a Research Article to the MBio Journal

Running title: "Metatranscriptomic analysis in acidophilic communities"

Abstract word count: 246 and 100. Manuscript word count: 5075. 


\section{ABSTRACT}

Gene expression profiles provide insight into how microorganisms respond to changing

3 environmental conditions. However, few studies have integrated expression profile analyses of

4 both coding genes and non-coding RNAs (ncRNAs) to characterize the functional activity of

5 microbial community members. Here, we defined gene expression profiles from environmental

6 and laboratory-grown acidophilic biofilms using RNASeq. In total, 15.8 million Illumina reads

7 were mapped to the genomes of 26 acidophilic microorganisms and nine viruses reconstructed

8 from the Richmond Mine at Iron Mountain, California. More than 99\% of the genome was

9 transcribed in three Leptospirillum species, and $>80 \%$ in the archaea G-plasma and Ferroplasma

10 Type II. High gene expression by G-plasma and the Leptospirillum Group II UBA strain

11 correlated with extremely acidic conditions, whereas high transcriptional expression of

12 Leptospirillum Group III and Leptospirillum Group II 5way-CG strain occurred under higher $\mathrm{pH}$

13 and lower temperature. While expression of CRISPR Cas genes occurs on the sense strand,

14 expression of the CRISPR loci occurs on the antisense strand in the Leptospirilli. A novel

15 riboswitch associated with the biosynthetic pathway for the osmolyte ectoine was upregulated

16 when each specific Leptospirillum Group II strain was growing under the conditions most

17 favorable for it. Newly described ncRNAs associated with CO dehydrogenase (CODH) suggest

18 regulation of expression of $\mathrm{CODH}$ as a $\mathrm{CO}$ sensor in mature biofilms in the Leptospirilli. Results

19 reveal the ways in which environmental conditions shape transcriptional profiles of organisms

20 growing in acidophilic microbial communities and highlight the significance of ncRNAs in

21 regulating gene expression. 


\section{IMPORTANCE}

23 Microorganisms play important roles in environmental acidification and in metal-

24 recovery based bioleaching processes. Therefore, characterizing how actively growing microbial

25 communities respond to different environments is key to understanding their role in those

26 processes. Microorganisms express their genes, both coding and non-coding, differently

27 depending on environmental factors, thus evaluating community expression profiles inform

28 about the ecology of actively growing microorganisms. Here we used community transcriptomic

29 analyses to characterize gene expression profiles from biofilm communities growing under

30 extremely acidic conditions. Results expand our knowledge of how acidophilic microorganisms

31 respond to changes in their environment and provide insight into possible gene regulation

32 mechanisms.

34 INTRODUCTION

35 Extremely acidic environments are usually dominated by relatively few taxa making them

36 good model systems for ecology and physiology studies. Because of the roles acidophilic

37 microorganisms play in environmental acidification and in metal-recovery based bioleaching

38 processes, gene expression studies are key to understanding the physiology and ecology of

39 microorganisms in acidic environments. The Richmond Mine at Iron Mountain, California, is a

40 well-studied acid mine drainage (AMD) system: for example, deep sequencing of many

41 Richmond Mine biofilms has enabled reconstruction of the genomes of many Bacteria, Archaea,

42 viruses, plasmids, and fungus (e.g. (1-4)). Genome reconstruction analyses, along with high-

43 throughput transcriptomic sequencing and mass spectrometry-based proteomics have the

44 potential to provide information about the community composition and activity in natural

45 ecosystems. Community transcriptomic analyses have been used to describe important metabolic 
46 processes in natural communities such as nitrogen metabolism in marine environments (5), the

47 flow of carbon between organisms in photosynthetic microbial mats (6), and associations of

48 microbial communities with the biogeochemistry of permafrost thawing environments (7). In

49 acidic environments, mass spectrometry-based community proteomics measurements of the

50 dominant member of Richmond Mine AMD biofilms indicated that biofilm maturation affects

51 Leptospirillum group II protein expression profiles, with the most protein expression variation

52 found as biofilms transition from early and mid-developmental to the most mature growth stage

53 (8). Similarly, microarray-based community transcriptomics indicated that expression profiles of

54 genes involved in biofilm formation, chemotaxis, motility and quorum-sensing in Leptospirillum

55 ferrooxidans were up-regulated when living as part of biofilms vs. in the planktonic fraction of

56 the Rio Tinto AMD system (9). Hua et al. recovered the genome of Ferrovum spp. from an acid

57 mine drainage system and community gene expression profiles indicated active $\mathrm{CO}_{2}$ fixation and

58 sulfate reduction pathways (10). In addition, metatranscriptomics studies have highlighted the

59 importance of non-coding RNA (ncRNA) expression in marine systems (11). However, studies

60 of the transcriptional profiles of coding and ncRNAs in acidophilic communities relevant for

61 AMD generation and bioleaching-based metal recovery have not yet been reported.

62 Many ncRNAs have been identified in Bacteria and Archaea, such as riboswitches,

63 ribozymes, and other regulatory ncRNAs, which play important roles in regulating gene

64 expression (reviewed in (12)). Riboswitches are regions within a messenger RNA (generally

65 located in the 5' untranslated region - UTR) containing ligand-binding sensors that regulate

66 downstream coding sequences (reviewed in (13)). Other regulatory ncRNAs with known

67 function include the tmRNA, which helps unlock stalled ribosomes, and the 6S ncRNA, which

68 acts as mimic of a promoter (reviewed in (14)). In Eukaryotes, a common mechanism of gene

69 regulation involves transcription of long 5' UTRs with secondary structures that can influence 
70 transcription of downstream genes, or containing short upstream open reading frames (uORFs)

71 that attenuate translation of the downstream protein (reviewed in (15)). In addition, high-

72 throughput transcriptomics analyses have enabled identification of ncRNAs that regulate

73 antibiotic resistance in bacteria via 5' UTR transcription and uORF attenuation (reviewed in

$74(16))$

75 Here we report the analyses of transcriptional profiles of non-ribosomal RNA from

76 biofilms collected from the Richmond Mine at Iron Mountain, California, and from laboratory-

77 grown biofilm communities. Gene expression profiles were evaluated for the whole acidophilic

78 community including Bacteria, Archaea, and viruses, and novel ncRNAs were discovered for

79 many community members. The results greatly expand our understanding of the responses of

80 acidophilic organisms to changes in their environment and provide insight into possible gene

81 regulation mechanisms by ncRNAs in the Leptospirilli.

82 (Part of this article was submitted to an online preprint archive (17))

84 RESULTS

85 Transcriptomics reads map across the whole genome of AMD community members.

86 On average $91.82 \% \pm 4.35 \%$ of the transcriptomic reads in total RNA samples and $8779.80 \% \pm 9.59 \%$ in the rRNA-subtracted samples mapped to rRNA genes from the SSU and LSU

88 Silva databases (Table 1). Despite the low efficiency of the rRNA-depletion protocol used, deep

89 sequencing allowed us to detect transcripts from 37 near-complete and partial genomes of

90 Bacteria, Archaea, Fungi, plasmids and viruses (Supplementary Table S1). Transcriptomic data

91 indicate that Leptospirillum group II C75 and UBA genotypes dominate early and mid-

92 developmental stage environmental biofilms characterized by extremely low $\mathrm{pH}$ and high

93 temperature, whereas Leptospirillum group II 5way-CG is the most abundant genotype in the late 
94 developmental-stage environmental biofilm (Figure 1A). Leptospirillum group III bacteria

95 dominate bioreactors and the A-drift environmental sample. Leptospirillum ferrooxidans and

96 Acidithiobacillus caldus were detected at very low abundance, as were other organisms and

97 viruses, some of which represent less than $0.001 \%$ of the community transcriptome

98 (Supplementary Table S1). Low Archaeal gene expression and high Acidithiobacillus sp.

99 expression have been documented on acid mine drainage biofilms growing at higher $\mathrm{pH}$ (18).

100 Transcriptomic reads spanned $>95 \%$ of the genome of Leptospirillum group II and group

101 III, the G-plasma archaeon and one viral genome (Figure 1B). In addition, up to 80\% of the

102 genome of Ferroplasma Type II, and over 60\% of the genomes of Ferroplasma Type I and three

103 viruses were identified as transcribed in some samples (Figure 1B). Likely, higher sequencing

104 coverage and/or higher rRNA-removal efficiency would support detecting whole genome

105 transcription in low-abundance community members. The results suggest that for dominant and

106 low-activity organisms and viruses the whole genome is transcribed at some level. This trend has

107 been observed previously in transcriptomics analyses of Bacillus anthracis isolates (19).

\section{Gene expression profiles from AMD taxa correlate with the environment $\mathbf{p H}$.}

110 Mapping reads to the predicted genes of AMD organisms, plasmids, and viruses were

111 assembled to yield over 26,300 transcripts (Supplementary Materials - R data). Differential

112 expression analysis identified $>4,800$ significantly differentially expressed genes with a false

113 discovery rate of 0.05 when evaluating environmental vs. bioreactor-grown biofilms

114 (Supplementary Figure S1A). Non-metric multidimensional scaling (NMDS) ordination on the

$115 \sim 4,800$ differentially expressed genes indicates two main axes responsible for most of the sample

116 variation (Figure 2, and Supplementary Figure S1B). The NMDS samples plot shows that axis 1

117 separates primarily based on environment type, while axis 2 separates based on pH (Figure 2A). 
118 Gene expression profiles from Leptospirillum group II UBA and C75 genotypes appear

119 correlated with negative NMDS2 scores (low $\mathrm{pH}$ environments), while expression profiles from

120 Leptospirillum group II 5way-CG genotype and group III appear correlated with positive

121 NMDS2 scores (higher pH environments) (Figure 2B-D). Gene expression profiles from Archaea

122 and viruses generally correlated with positive NMDS1 scores, that is, from environmental rather

123 than bioreactor-grown biofilms. Overall, the NMDS genes plots show a gradient of genes from

124 bacteria living at very low $\mathrm{pH}$ and high temperature (Figure 2B, bottom right quadrant),

125 transitioning towards genes from Archaea, viruses and other low-abundance organisms (Figure

126 2C-D), and ending on genes from bacteria living in bioreactors at higher $\mathrm{pH}$ and lower

127 temperature (Figure 2B, top left quadrant). Results indicate that environmental gradients directly

128 affect the transcriptional profiles of the community members living along them. Gradients of $\mathrm{pH}$,

129 temperature, and dissolved oxygen, among others, have been suggested as factors influencing

130 community assembly in acidic environments $(20,21)$.

131 Environment-specific transcriptional profiles are also evident from hierarchical clustering

132 of transcribed genes with a $\log 2$-fold change $>5$, which separates samples into environmental

133 and bioreactor biofilms, and environmental samples appear to also cluster by developmental

134 stage (Supplementary Figures S1C and S1D). For example, Leptospirillum group II 5way-CG

135 and Leptospirillum group III genes are overrepresented in the bioreactors and in the A-drift

136 environmental sample (Supplementary Figures S1D). The A-drift biofilm was collected from a

137 very oxidized pool, at higher $\mathrm{pH}$ and lower temperature than other environmental samples (see

138 Table 1), conditions that appear to favor growth of these organisms in the laboratory bioreactors.

139 Genes from Leptospirillum group II UBA are over-represented in early to mid-growth-stage

140 environmental biofilms relative to bioreactor-grown biofilms (Supplementary Figure S1D). This

141 genotypic group is also well represented in biofilms collected from the C-drift locations, many 
142 of which have reported very low $\mathrm{pH}$ and high temperature environments (22-24). Our results

143 support previous proteomic-based studies that suggested distinct ecological adaptation of two

144 Leptospirillum group II strains (22). Leptospirillum group II-associated AMDV1 phage genes,

145 as well as genes from the AMDV3 virus are overrepresented in the environmental biofilms, while

146 unassigned-viral genes (AMDVIR, likely Leptospirillum-associated phage) are overrepresented

147 in bioreactors (Supplementary Figure S1C and Figure 2D). Results indicate that phage/virus

148 activity correlates with their host gene expression.

149 When looking at the transcriptional profiles of the most abundant community members,

150 hierarchical clustering of expressed genes indicates that genes involved in energy production and

151 conversion, carbon fixation, fatty acid metabolism, transcription and translation factors, and

152 ribosomal proteins from Leptospirillum group II UBA and C75 are highly expressed in early to

153 mid-growth-stage environmental biofilms (Figure 3A-B, 3E). These findings suggest rapid

154 growth during early and mid-successional stages. Genes generally overrepresented in bioreactor

155 samples include those involved in amino acid and cofactor metabolism, carbohydrate and lipid

156 metabolism, DNA repair and recombination, lipopolysaccharide metabolism, nucleic acid

157 metabolism, signal transduction, tRNA synthetases and transport genes from Leptospirillum

158 group II 5way-CG and group III (Figure 3C-D and 3E). Bioreactors usually have higher pH and

159 lower temperature than most environmental biofilms from the Richmond Mine (Table 1). These

160 conditions might slow down the activity of Leptospirillum group II UBA and C75, and of G-

161 plasma, while enriching for growth and transcriptional activity of Leptospirillum group III and

162 group II 5way-CG.

163

164 Regulatory motifs, mobile elements and expression of ncRNAs of known function. 
Inspection of the upstream regions of transcribed coding genes, tRNAs and non-coding

166 RNAs predicted 207 RpoD promoters in the Leptospirillum group II UBA genotype

167 (Supplementary Table S2): $11.5 \%$ of them were associated to coding genes or operons

168 transcribed in at least 10 of the 13 biofilm samples. Promoter length varies between 8 and 33 bp

169 and a sequence logo (25) representation of the overall promoter structure indicates that most

170 promoters contain a -10 and -35 motif (Figure 4A).

171 Transposases in Leptospirillum group II UBA and group III are among the most highly

172 expressed genes (Supplementary Materials - R data). In addition, highly expressed multi-copy

173 transposases are enriched in environmental than bioreactor samples (Supplementary Figure S2).

174 Highly abundant transposase expression was reported in community proteomic analyses in acid

175 mine drainage biofilms (26), and in community transcriptomic analyses $(27,28)$. It is possible

176 that the movement of mobile elements is very important in natural acidophilic biofilms.

177 A few of the well-characterized ncRNAs in the Rfam database were identified in many

178 genomes of acidophilic microorganisms, some of which are among the most highly expressed

179 genes (Supplementary Table S3 and Figure 4). SsrA (aka transfer-messenger RNA or tmRNA)

180 was detected in the Leptospirilli (Figure 4B-C), as well as in G-plasma, a plasmid, and in

181 Acidithiobacillus caldus, the later representing only $0.003 \%$ of the community transcriptome in

182 the sample in which it is most abundant (Adrift GS0, Supplementary Table S1). The RNAse P

183 ncRNA was the most highly expressed ncRNA in the transcriptomic datasets, and was identified

184 in most AMD organisms, including the fungus, the very low abundance $A$. caldus and $L$.

185 ferrooxidans bacteria, and the archaeon C-plasma (Supplementary Table S3). The signal

186 recognition particle (SRP) RNA was also highly expressed in the Leptospirilli (Figure 4B-C),

187 Ferroplasma Type I and Type II, the fungus, and in G-, A-, E- and C-plasma. The 6S RNA (SsrS)

188 was identified in the Leptospirilli (Figure 4B-C), the Sulfobacillus, and A. caldus. The cobalamin 
189 riboswitch was only identified in Leptospirillum group III and contains the expected conserved

190 domains within the core region as described in other organisms (29) (Figure 4C). The cobalamin

191 riboswitch has not yet been described in the genomes of any other member of the Nitrospira

192 Phylum.

Among the ncRNAs associated to mobile elements detected in the transcriptomes are the

194 Group II introns of Leptospirillum groups II and III, Ferroplasma Type II, and the

195 Actinobacterial Bin 1 (Supplementary Table S3). In addition, the 5' UTR region of several HNH-

196 endonucleases in Leptospirillum groups II, III and IV, and Ferroplasma Type II contain the non-

197 coding HEARO RNA, which, along with its associated endonuclease, constitute another type of

198 mobile genetic element (30). The hgcC RNA, a non-coding RNA of unknown function, was

199 detected in Leptospirillum group II UBA and group II 5way-CG, and in two viruses

200 (Supplementary Table S3). Previously the hgcC RNA was reported only in Archaea (31),

201 although it is also found in a few viruses in the Rfam database.

202 The small nucleolar RNA (snoRNA), a eukaryotic small RNA with few known homologs

203 in Archaea (32) was detected in most Richmond Mine Archaea A-, C- and G-plasma,

204 Ferroplasma Type I and Type II, and the fungus; while the crcB RNA (a fluoride riboswitch,

205 (33)) was detected in the Leptospirilli, G-plasma, and in Ferroplasma Type II (Supplementary

206 Table S3).

207 The TPP riboswitch binds thiamine pyrophosphate to regulate expression of thiamine

208 biosynthesis and other related genes. These riboswitches have been identified upstream of multi-

209 transmembrane hypothetical proteins in the genomes of Thermoplasma acidophilum,

210 Thermoplasma volcanicum, and Ferroplasma acidarmanus (34). A-, G-, and E-plasma

211 (members of the Thermoplasmatales), I-plasma and Ferroplasma Type II express the TPP 
212 riboswitch and reads within it have paired-reads to a downstream putative transporter likely

213 regulating its expression (Supplementary Table S3).

215 CRISPR Cas genes and loci expression occurs on both strands of DNA.

216 Transcripts from CRISPR-associated Cas genes were detected in all samples for

217 Leptospirillum groups II and III, the archaeon Ferroplasma Type I, and Archaea A- and G-

218 plasma (Supplementary Table S4). Cas genes from Leptospirillum group II UBA and G-plasma

219 showed higher expression in bioreactor samples, while those from Leptospirillum group III and

220 group II 5way-CG showed higher expression in environmental biofilms (Figure 3E).

221 When surveying the transcriptomic reads using the CRASS software, transcripts

222 containing CRISPR repeats from eight datasets were detected in Leptospirillum groups II and III,

223 G-plasma, Ferroplasma Type I and Type II, the Actinobacterial bin, and plasmids

224 (Supplementary Tables S4 and S5). Some additional CRISPR transcripts could not be assigned

225 to an AMD genome based on known repeat sequences. The highest diversity of CRISPR repeats

226 expressed was observed in the late growth-stage biofilm (4-way_GS2; Supplementary Table S4).

227 This finding probably reflects the higher richness of late growth-stage biofilms as well as the

228 activity of multiple closely related strains with slightly different CRISPR loci (and thus different

229 phage/viral susceptibility). For Leptospirillum group II and G-plasma, the largest number of

230 distinct spacer transcripts was detected in this same biofilm, consistent with a higher diversity of

231 strains in the sample.

232 Transcriptomic reads from precursor CRISPR RNA (pre-crRNAs, as reviewed in (35)) aligned

233 mostly at the trailer end (containing older CRISPR spacers) of the composite CRISPR loci from

234 the assembled genome of Leptospirillum group II UBA (Figure 5A), and no repeats and spacer-

235 carrying reads aligned to the CRISPR loci from the assembled Leptospirillum group III genome. 
236 These observations are likely due to the composite assemblies not capturing the dynamics of

237 acquired spacers from closely related strains. For example, 407 of the 688 different spacer 238 sequences recovered from Leptospirillum group II transcripts, and 5 of the 245 spacers detected

239 in the transcriptome in Leptospirillum group III (Supplementary Table S5) had been recovered

240 in a previous analysis of Richmond Mine CRISPR systems (36). Notably, while transcription of

241 Cas genes occurs on the sense strand in which the genes were predicted, transcription of the

242 CRISPR loci (repeat/spacer region) in Leptospirillum group II UBA occurs on the antisense

243 strand (Figure 5A). Moreover, 4 of the 5 Leptospirillum group III CRISPR spacers and 404 of

244 the 407 spacers in Leptospirillum group II UBA recovered here via strand-specific

245 transcriptomics represent the reverse complement of the sequences previously reported in (36)

246 (Supplementary Table S5).

248 Expression of novel ncRNA.

249 We observed transcription of long leader sequences upstream of many genes and operons

250 in Leptospirillum groups II and III. One example is a transcribed $370 \mathrm{bp}$ 5' UTR of the ectoine

251 biosynthesis operon in both Leptospirillum group II UBA and 5way-CG strains (Figure 5B-C).

252 Ectoine is a compatible solute either synthesized or transported from the environment by many

253 organisms during osmotic stress $(37,38)$. The length of the ncRNA upstream of the ectoine

254 operon, the presence of paired reads from the ncRNA to the transcribed operon, and the presence

255 of a putative promoter (TTGACA-N17-(A)A(A)A(C)T), a Rho-independent terminator (-6.30

$256 \mathrm{Kcal} / \mathrm{mol})$ and an anti-terminator $(-7.03 \mathrm{Kcal} / \mathrm{mol})$ suggest it is likely a riboswitch. The

257 expression of the putative riboswitch in Leptospirillum group II UBA in two environmental

258 biofilms is generally higher than that of the operon (Figure 5B, top panel, green and red curves).

259 However, the transcript levels of the putative riboswitch in the bioreactor samples are much lower 
260 than those of the operon (Figure 5B, top panel, blue and magenta curves). These results suggest

261 that the riboswitch may inhibit transcription of the operon in the environmental samples

262 (preferred conditions for Leptospirillum group II UBA growth, see Figure 1) and enhance the

263 ectoine operon transcription in bioreactor samples (where growth conditions are more stressful).

264 The expression of the putative riboswitch in Leptospirillum group II 5way-CG shows a slight

265 opposite trend than in Leptospirillum group II UBA (Figure 5B, bottom panel). The riboswitches

266 of both bacteria share $80 \%$ identity at the nucleotide level and their predicted secondary structures

267 look very different (Figure 5C).

268 Novel carbon monoxide dehydrogenase-associated ncRNAs (referred to here as CODH-

269 ncRNA) were identified in long transcribed leader sequences in Leptospirillum group III and in

270 Leptospirillum groups II UBA and 5way-CG genotypes (Figure 6 and Supplementary Figure S3).

271 The CODH-ncRNA is expressed at similar abundance and contains paired-end reads that map to

272 its downstream CO-dehydrogenase beta subunit gene (the only subunit identified in the

273 Leptospirilli). Although terminators could not be predicted, the dip in the transcriptional

274 expression of the CODH-ncRNAs before the ribosome binding site (RBS) of their associated

275 CO-dehydrogenase $(\mathrm{CODH})$ genes suggest that the CODH-ncRNA may be regulating CODH

276 expression (Figure 6 and Supplementary Figure S3). Although the Archaeon Ferroplasma Type

277 II has multiple copies of the full $\mathrm{CODH}$ operon and all subunits showed transcriptional

278 expression, a CODH-ncRNA was not identified upstream of the operons (data not shown). Two

279 of the three copies of the CODH-ncRNA in Leptospirillum group III and both copies in

280 Leptospirillum group II are highly expressed in the late developmental-stage biofilms (Figure 6,

281 left panel; Supplementary Figure S3A-D).

282 We observed expression of many long leader sequences upstream of other important

283 coding genes and operons in Leptospirillum groups II and III. For example, the transcribed 5' 
284 UTR of the pyruvate ferredoxin oxidoreductase (PFOR) operon, cytochrome 572 and

285 cytochrome 579 genes contain putative promoters and untranslated ORFs (uORFs) (Figure 6 and

286 Supplementary Figure S3E-I). Other examples include expression of the 5' UTR of the

287 Uroporphyrin-III C-methyltransferase gene and of the Ribonuclease III gene in Leptospirillum

288 group II UBA, with potential folded ncRNAs and predicted promoters (Supplementary Figure

289 S3J-K).

\section{DISCUSSION}

Community expression profiles inform about the ecology and physiology of actively

293 growing organisms in their natural environment. Richmond Mine AMD biofilms develop in

294 extremely acidic conditions and their characterization is vital to understanding the process of

295 water acidification. Bioreactor conditions generally resemble environmental conditions from

296 downstream AMD sites at higher $\mathrm{pH}$ and lower temperatures, as well as those from metal-release

297 bioleaching systems. Therefore, analyses of environmental and laboratory-grown acidophilic

298 biofilms improve our understanding of the dynamics occurring in acid drainage and bioleaching

299 ecosystems.

300 Acid mine drain communities have been extensively studied for community membership,

301 and proteomic and transcriptomic studies have been used to infer ecological roles of community

302 members (reviewed in (20)). However, no reports have analyzed the transcriptional profiles of

303 both coding genes and non-coding RNAs (ncRNAs) in acidophilic communities. Here,

304 metatranscriptomic analysis of natural AMD biofilms and of acidophilic biofilms growing in

305 laboratory bioreactors identified patterns in gene expression that correlate with organismal

306 environmental preferences and reveal the potential roles of known and novel ncRNAs. 
Differential gene expression analyses indicated that preferred growth conditions for

308 Leptospirillum group II UBA and C75 genotypes, and the Archaeon G-plasma are environments

309 at very low $\mathrm{pH}$ and high temperatures, while Leptospirillum group II 5way-CG and group III

310 prefer higher $\mathrm{pH}$ and lower temperature conditions. AMD organisms appear to show base-level

311 activity by expressing the whole genome, and Leptospirillum-associated viruses follow

312 transcriptional expression profiles similar to that of their host. In addition, gradients of $\mathrm{pH}$ and

313 temperature evident from differentially expressed genes, high expression of transposase genes,

314 and the high diversity of CRISPR loci spacers suggest that AMD communities are highly

315 dynamic ecosystems.

316 It has been suggested that Cas proteins and CRISPR loci primary transcripts are

317 constitutively expressed, and their expression levels might be induced as invasion occurs

318 (reviewed in (39)). Differential expression of CRISPR Cas genes in environmental vs. bioreactor

319 biofilms point to some level of regulation, where Cas genes are up-regulated in less optimal

320 environments for the organisms carrying them. Leptospirillum Group II Cas proteins, at the time

321 considered hypothetical, were highly abundant in the first microbial community proteomic

322 analysis (26) and expression of Cas genes was observed previously in Leptospirillum group III

323 (18). When examining the strand-specific nature of the transcriptome, we observed that CRISPR

324 loci (repeats and spacers) transcription occurs on the antisense strand while Cas genes are

325 expressed on the sense strand in the Leptospirilli. Co-expression of sense and antisense mRNAs

326 in plants was shown more effective at offering viral immunity than only sense or antisense

327 expression of the relevant genes (40). Therefore, Cas genes and CRISPR loci expression on

328 different strands may be used as a mechanism for effective viral immunity in the Leptospirilli.

329 Although, differential expression of Cas genes and CRISPR loci on opposite strands has not yet

330 been reported, bidirectionality of CRISPR loci transcription was observed in Sulfolobus sp., 
331 where the authors suggest the potential need for bidirectionality to neutralize the leader spacer

332 RNAs in the absence of invading elements (41).

RNASeq analyses enable identification of regulatory ncRNAs, leader sequences and

334 transcription start sites (TSS) for coding genes and operons. We confirmed expression of

335 ncRNAs of known function in many high and low abundance organisms, including the RNAse

$336 \mathrm{P}$, SsrA and 6S ncRNAs previously predicted in silico in Acidithiobacillus caldus (42). In

337 addition, some ncRNAs of unknown function and 5' UTRs show expression levels similar to

338 their neighboring genes and predicted operons, suggesting that expression of upstream non-

339 coding regions might be regulating their neighboring genes. For example, we identified a novel

340 riboswitch associated to the ectoine biosynthetic pathway in Leptospirillum group II. The

341 riboswitch appears to inhibit transcription of the ectoine operon in favorable growth conditions

342 while enhancing transcription during stress, suggesting that Leptospirillum group II synthesis of

343 compatible solutes to tolerate unfavorable conditions is regulated by the ectoine riboswitch.

344 Other novel regulatory ncRNAs include a carbon monoxide dehydrogenase-associated

345 ncRNA (CODH-ncRNA) in Leptospirillum group II and III. CO dehydrogenase, a nickel iron-

346 sulfur protein, is usually part of a multi-protein complex which metabolizes carbon monoxide

347 (CO) when the cells sense $\mathrm{CO}$ in the environment, as well as being used as electron and carbon

348 sources by some bacteria (reviewed by (43)). The CODH beta-subunit, the only subunit identified

349 in Leptospirillum groups II and III, has been reported as a single active subunit in metal-reducing

350 Geobacter bacteria and in the genomes of two Chlorobium phototrophic bacteria (44). It is highly

351 sensitive to oxygen, which might explain the high expression of both CODH and CODH-ncRNA

352 transcripts in late developmental stage biofilms. It is possible that the CODH-ncRNA up-

353 regulates expression of the $\mathrm{CO}$ dehydrogenase gene as biofilms mature. 
Long transcribed 5'UTRs with putative upstream ORFs (uORFs) preceding energy-

355 generation genes (e.g. PFOR and cytochrome c) likely regulate transcription and/or translation

356 of their downstream genes and operons (Figure 6). Analysis of transcription start sites (TSS) in

357 Clostridium phytofermentans have shown that the TSS of one pfor gene occurs 78 bp upstream

358 of its start codon (45), a length similar to the putative TSS of the pfor operons in Leptospirillum

359 sp. (Figure 6), while transcripts with long 5' UTRs encoding putative ncRNAs and uORFs have

360 been reported in Haloferax volcanii (46) and in Mycobacterium sp. (47). Overall, our results

361 point to significant roles of regulatory ncRNAs in acidophilic communities and suggest that gene

362 expression profiles appear to be a factor in ecological diversification.

\section{METHODS}

365 Eight biofilms at different developmental stages (growth-stage (GS) 0, 0.5, 1, and 2) were

366 collected from the A-, C-, AB-drift, and 4way locations within the Richmond Mine at Iron

367 Mountain Mines, California (4040' 38.42" $\mathrm{N}$ and 122" 31' 19.90" W, elevation of approx.

$368900 \mathrm{~m})($ Table 1). AMD biofilm developmental stages were estimated visually based on thickness

369 (23). In addition, biofilms were grown in the dark, at $\mathrm{pH} 1$ and $37^{\circ} \mathrm{C}$ in laboratory bioreactors, 370 as described in (48) using inocula from within the A-drift location and mine drainage outflow

371 (Table 1). Biofilms were harvested when reaching growth-stage 0, 0.5, and 1, snap-frozen in 372 liquid nitrogen upon collection/harvest and stored at $-80{ }^{\circ} \mathrm{C}$.

373 Total RNA was extracted from frozen samples using two acid phenol-chloroform-

374 isoamyl alcohol extractions and immediately purified using the RNEasy MinElute kit (Qiagen).

375 Ribosomal RNA (rRNA) subtraction on 8 of the 13 biofilm samples was done using the

376 MicrobExpress kit (Ambion). Good quality RNA (RIN > 7, assessed by a Bioanalyzer 2100

377 (Agilent Technologies)) from total RNA and rRNA-subtracted RNA were converted to cDNA as 
378 described by (49) in order to keep the strand-specificity of the transcriptome. Briefly, the

379 technique involves adding deoxy-UTP in place of deoxy-TTP during synthesis of the second

380 strand of cDNA. After Illumina library preparation, the second strand of cDNA is selectively

381 digested allowing for sequencing of all molecules in the same direction, and sequences maintain

382 the strand-specificity of the original RNA molecules (49). Resulting cDNA was fragmented using

383 a Covaris S-system (Covaris, Inc.) to an average fragment size of $200 \mathrm{bp}$ and sent to the

384 University of California Davis for Illumina library preparation, digestion of the dUTP-containing

385 strand, and sequencing. Five samples were sequenced using the GAIIx platform (75 bp, single

386 end reads), while eight samples were sequenced using the HiSeq 2500 platform (100 bp, paired-

387 end reads) (Table 1).

Low-quality bases were trimmed from the sequencing reads using the fastx_trimmer

script (http://hannonlab.cshl.edu/fastx_toolkit/) and the sickle trimmer script with default

390 parameters (https://github.com/najoshi/sickle) and reads $<40$ bp in length were discarded.

391 Trimmed reads were mapped to the SSU and LSU rRNA gene Silva database SSURef_102 (50)

392 using Bowtie2 (51) with default parameters to separate ribosomal from non-ribosomal reads.

393 Non-ribosomal (non-rRNA) reads were mapped using Bowtie2 with default parameters

394 to the available genomes of acidophilic bacteria: Leptospirillum ferrooxidans C2-3,

395 Leptospirillum group II UBA type (L. rubarum), group II '5way-CG' type, group II 'C75' type,

396 group III (L. ferrodiazotrophum), group IV UBA BS, Acidithiobacillus caldus ATCC 51756, a

397 Sulfobacillus bin, and two Actinobacterial bins (2, 3, 22, 52-56); archaea: A, C, D, E, G, and I-

398 plasma, ARMAN-1, -2, -4, and -5, and Ferroplasma Type I and Type II (2, 4, 57-59); plasmids

399 (53), a fungus (1); and nine viruses/phage (60). Mapped reads were then assembled into transcript

400 fragments using the Cufflinks pipeline (61). The genome references for mapping are available

401 at: http://genegrabber.berkeley.edu/amd/organisms. 
402 The relative abundance of genomes and genomic fragments from AMD organisms in each sample

403 was estimated as the coverage for all transcripts within a genome (read counts were normalized

404 for genome length and total number of reads per sample) (Figure 1 and Supplementary Table

405 S1). Assembled transcripts from all organisms were also searched vs. the Rfam database (62) for

406 non-coding RNAs (ncRNA) of known function. Additionally, the transcriptional profiles of

407 Leptospirillum groups II UBA, group II 5way-CG, group III, and of the archaea A-plasma, C-

408 plasma, G-plasma, and Ferroplasma Type II, were visually inspected using Artemis (63).

409 Transcribed regions that did not fall within a coding sequence were evaluated for the presence of

410 non-annotated protein sequences using BlastX (64) vs. the non-redundant NCBI database. We

411 scanned these regions for the presence of possible ribosome binding sites and start codons that

412 could hint to hypothetical proteins not yet identified in the public databases. Transcribed

413 intergenic regions that do not appear to encode for protein sequences, based on the above criteria,

414 were labeled as potential non-coding RNA (ncRNA). Bacterial promoters were predicted

415 upstream of manually evaluated ncRNAs using BPROM (65) and rho-independent terminators

416 were evaluated with ARNOLD (66). Riboswitch motifs were predicted using the RibEx

417 webserver (67), and secondary structure prediction of the ncRNAs was done using the RNAfold

418 webserver (68).

419 Correlation analyses of assembled transcript abundances of non-rRNA reads that mapped

420 to the predicted genes of AMD organisms were done on samples for which total and rRNA-

421 depleted reads were obtained (Supplementary Figure S4). Transcript abundances correlated well

422 for most samples ( $\mathrm{R}^{2}$ values range from 0.74 to 0.95 ) hence, non-rRNA reads from rRNA-

423 depleted RNA were pooled with those non-rRNA reads from their corresponding total RNA. The

424 correlation between rRNA-depleted and total RNA transcripts from an early developmental stage

425 bioreactor sample, R1_GS0, was low (and dispersion of points was high), likely due to the much 
426 shorter fragments assembled from the total RNA sample (Supplementary Figure S4H). Given

427 that the correlation between transcript abundances was slightly positive, and that transcript length

428 improved in the rRNA-depleted sample, reads from total and rRNA-depleted RNA were also

429 pooled for R1_GS0.

431 (69). Read counts were normalized for gene length using the function

432 “withinLaneNormalization” from the EDASeq package (70). NMDS ordination was performed

433 on Bray-Curtis distance matrices of variance-stabilizing transformed gene abundance tables with

434 the formula: genes sequencing type + environment type, using the DESeq2 R package (71).

435 Putative RpoD promoters were predicted upstream of expressed genes in Leptospirillum

436 group II UBA genotype by manual inspection of the genome using Artemis (63) along with the

437 visualized transcriptome data. Predicted promoters were listed if they were less than 50 bp

438 upstream of the 5 ' end of the mapped reads of a transcript and matched 8 out of 12 bp of the

439 standard E. coli / B. subtilis RpoD motif(TTGACA_N16-19_TATAAT).

440 Hierarchical clustering of abundances from predicted genes was done using the software

441 Cluster 3.0 for Mac OSX, centering genes and samples by the median, using the Spearman Rank

442 Correlation similarity matrix, and Average Linkage as clustering method (72). Clusters and heat

443 maps were visualized using the Java TreeView software (73). Gene trees for Leptospirillum spp.

444 transposases were constructed using the MABL website (74). CRISPR loci from transcriptomics

445 reads were reconstructed using the CRASS program (75).

447 DATA AVAILABILITY 
448 Raw sequencing reads were submitted to the NCBI sequence reads archive (SRA) under

449 accession number SRP026490 (76). R code and data tables are available at

450 https://github.com/dgoltsman/acid-mine-drainage.

451

452 ACKNOWLEDGEMENTS

453 We thank the late TW Arman, President of Iron Mountain Mines Inc., Mr R Sugarek (US

454 Environmental Protection Agency) for site access, and Mr R Carver for on-site assistance. This

455 project was funded by the U.S. Department of Energy, through the Carbon-Cycling (DE-FG02-

456 10ER64996) program.

457

458 


\section{REFERENCES}

460 1. Mosier AC, Miller CS, Frischkorn KR, Ohm RA, Li Z, LaButti K, Lapidus A, Lipzen A, 461 Chen C, Johnson J, Lindquist EA, Pan C, Hettich RL, Grigoriev IV, Singer SW, Banfield

462 JF. 2016. Fungi Contribute Critical but Spatially Varying Roles in Nitrogen and Carbon Cycling 463 in Acid Mine Drainage. Front Microbiol 7:238.

2. Dick GJ, Andersson AF, Baker BJ, Simmons SL, Thomas BC, Yelton AP, Banfield JF. 2009. Community-wide analysis of microbial genome sequence signatures. Genome Biol 10:R85.

3. Simmons SL, Dibartolo G, Denef VJ, Goltsman DS, Thelen MP, Banfield JF. 2008. Population genomic analysis of strain variation in Leptospirillum group II bacteria involved in acid mine drainage formation. PLoS Biol, 2008 ed. 6:e177.

4. Tyson GW, Chapman J, Hugenholtz P, Allen EE, Ram RJ, Richardson PM, Solovyev VV, Rubin EM, Rokhsar DS, Banfield JF. 2004. Community structure and metabolism through transcriptomics reveals universal patterns of protein sequence conservation in natural microbial communities. Genome Biol, 2011 ed. 12:R26. 
8. Mueller RS, Dill BD, Pan C, Belnap CP, Thomas BC, VerBerkmoes NC, Hettich RL,

Banfield JF. 2011. Proteome changes in the initial bacterial colonist during ecological succession in an acid mine drainage biofilm community. Environmental microbiology, 2011 ed. 13:22792292.

9. Moreno-Paz M, Gomez MJ, Arcas A, Parro V. 2010. Environmental transcriptome analysis reveals physiological differences between biofilm and planktonic modes of life of the iron oxidizing bacteria Leptospirillum spp. in their natural microbial community. BMC genomics,

10. Hua ZS, Han YJ, Chen LX, Liu J, Hu M, Li SJ, Kuang JL, Chain PS, Huang LN, Shu WS. metagenomics and metatranscriptomics. The ISME journal.

11. Shi Y, Tyson GW, DeLong EF. 2009. Metatranscriptomics reveals unique microbial small RNAs in the ocean's water column. Nature, 2009 ed. 459:266-269.

12. Wan Y, Kertesz M, Spitale RC, Segal E, Chang HY. 2011. Understanding the transcriptome through RNA structure. Nature reviews Genetics 12:641-655.

13. Serganov A, Nudler E. 2013. A decade of riboswitches. Cell, 2013 ed. 152:17-24. do, and how they do it. Adv Genet 90:133-208.

501 15. Hinnebusch AG, Ivanov IP, Sonenberg N. 2016. Translational control by 5'-untranslated regions of eukaryotic mRNAs. Science 352:1413-1416. Opin Microbiol 36:111-117. 
505 17. Goltsman DS. 2013. PhD thesis. Chapter 4. University of California, Berkeley. Permalink:

506 https://escholarship.org/uc/item/0zf9f3nx.

507 18. Chen L-X, Hu M, Huang L-N, Hua Z-S, Kuang J-L, Li S-J, Shu W-S. 2015. Comparative

508 metagenomic and metatranscriptomic analyses of microbial communities in acid mine drainage.

$509 \quad$ The ISME journal 9:1579-1592.

510 19. Passalacqua KD, Varadarajan A, Ondov BD, Okou DT, Zwick ME, Bergman NH. 2009.

511 Structure and complexity of a bacterial transcriptome. Journal of bacteriology, 2009 ed.

$512 \quad 191: 3203-3211$.

513 20. Quatrini R, Johnson DB. 2018. Microbiomes in extremely acidic environments: functionalities

514 and interactions that allow survival and growth of prokaryotes at low $\mathrm{pH}$. Current Opinion in

$515 \quad$ Microbiology 43:139-147.

516 21. Huang L-N, Kuang J-L, Shu W-S. 2016. Microbial Ecology and Evolution in the Acid Mine

517 Drainage Model System. Trends in Microbiology 24:581-593.

518 22. Denef VJ, Kalnejais LH, Mueller RS, Wilmes P, Baker BJ, Thomas BC, VerBerkmoes NC,

519 Hettich RL, Banfield JF. 2010. Proteogenomic basis for ecological divergence of closely related

520 bacteria in natural acidophilic microbial communities. Proceedings of the National Academy of

521 Sciences of the United States of America, 2010 ed. 107:2383-2390.

522 23. Wilmes P, Remis JP, Hwang M, Auer M, Thelen MP, Banfield JF. 2009. Natural acidophilic

523 biofilm communities reflect distinct organismal and functional organization. The ISME journal,

$524 \quad 2008$ ed. 3:266-270.

525 24. Mueller RS, Denef VJ, Kalnejais LH, Suttle KB, Thomas BC, Wilmes P, Smith RL,

526 Nordstrom DK, McCleskey RB, Shah MB, Verberkmoes NC, Hettich RL, Banfield JF. 2010. 
527 Ecological distribution and population physiology defined by proteomics in a natural microbial

528 community. Mol Syst Biol, 2010 ed. 6:374.

529 25. Schneider TD, Stephens RM. 1990. Sequence logos: a new way to display consensus sequences.

$530 \quad$ Nucleic acids research 18:6097-6100.

531 26. Ram RJ, Verberkmoes NC, Thelen MP, Tyson GW, Baker BJ, Blake RC2, Shah M, Hettich

532 RL, Banfield JF. 2005. Community proteomics of a natural microbial biofilm. Science, 2005 ed.

$533 \quad 308: 1915-1920$.

534 27. Frias-Lopez J, Duran-Pinedo A. 2012. Effect of periodontal pathogens on the

535 metatranscriptome of a healthy multispecies biofilm model. Journal of bacteriology, 2012 ed.

$536 \quad$ 194:2082-2095.

28. Hewson I, Poretsky RS, Beinart RA, White AE, Shi T, Bench SR, Moisander PH, Paerl RW,

Tripp HJ, Montoya JP, Moran MA, Zehr JP. 2009. In situ transcriptomic analysis of the globally important keystone N2-fixing taxon Crocosphaera watsonii. The ISME journal, 2009 ed.

29. Peselis A, Serganov A. 2012. Structural insights into ligand binding and gene expression control by an adenosylcobalamin riboswitch. Nature structural \& molecular biology, 2012 ed. 19:11823:618-631.

30. Weinberg Z, Perreault J, Meyer MM, Breaker RR. 2009. Exceptional structured noncoding RNAs revealed by bacterial metagenome analysis. Nature, 2009 ed. 462:656-659.

31. Klein RJ, Misulovin Z, Eddy SR. 2002. Noncoding RNA genes identified in AT-rich America 99:7542-7547. 
32. Omer AD, Lowe TM, Russell AG, Ebhardt H, Eddy SR, Dennis PP. 2000. Homologs of small nucleolar RNAs in Archaea. Science 288:517-522.

33. Breaker RR. 2011. Prospects for riboswitch discovery and analysis. Molecular cell, 2011 ed. 43:867-879.

34. Rodionov DA, Vitreschak AG, Mironov AA, Gelfand MS. 2002. Comparative genomics of thiamin biosynthesis in procaryotes. New genes and regulatory mechanisms. The Journal of biological chemistry, 2002nd ed. 277:48949-48959.

35. Marraffini LA, Sontheimer EJ. 2010. CRISPR interference: RNA-directed adaptive immunity in bacteria and archaea. Nature reviews Genetics 11:181-190.

36. Sun CL, Thomas BC, Barrangou R, Banfield JF. 2016. Metagenomic reconstructions of bacterial CRISPR loci constrain population histories. The ISME journal 10:858-870.

37. Empadinhas N, da Costa MS. 2006. Diversity and biosynthesis of compatible solutes in hyper/thermophiles. International microbiology : the official journal of the Spanish Society for

38. Saum SH, Muller V. 2008. Growth phase-dependent switch in osmolyte strategy in a moderate halophile: ectoine is a minor osmolyte but major stationary phase solute in Halobacillus halophilus. Environmental microbiology, 2007 ed. 10:716-726.

39. Bhaya D, Davison M, Barrangou R. 2011. CRISPR-Cas systems in bacteria and archaea: versatile small RNAs for adaptive defense and regulation. Annual review of genetics, 2011 ed. 45:273-297.

40. Waterhouse PM, Graham MW, Wang MB. 1998. Virus resistance and gene silencing in plants can be induced by simultaneous expression of sense and antisense RNA. Proceedings of the National Academy of Sciences of the United States of America 95:13959-13964. 
572 41. Lillestøl RK, Shah SA, Brügger K, Redder P, Phan H, Christiansen J, Garrett RA. 2009.

573 CRISPR families of the crenarchaeal genus Sulfolobus: bidirectional transcription and dynamic

$574 \quad$ properties. Molecular microbiology 72:259-272.

575 42. Shmaryahu A, Holmes DS. 2007. Discovery of Small Regulatory RNAs Extends Our

576 Understanding of Gene Regulation in the Acidithiobacillus Genus. Advanced Materials Research

$577 \quad 20: 535-538$.

578 43. Fontecilla-Camps JC, Ragsdale SW. 1999. Nickel-Iron-Sulfur Active Sites: Hydrogenase and

579 Co Dehydrogenase. Advances in Inorganic Chemistry 47:283-333.

580 44. Techtmann S, Colman AS, Lebedinsky AV, Sokolova TG, Robb FT. 2012. Evidence for

581 Horizontal Gene Transfer of Anaerobic Carbon Monoxide Dehydrogenases. Front Microbiol 3.

45. Boutard M, Ettwiller L, Cerisy T, Alberti A, Labadie K, Salanoubat M, Schildkraut I,

Tolonen AC. 2016. Global repositioning of transcription start sites in a plant-fermenting bacterium. Nature communications 7:13783.

46. Babski J, Haas KA, Näther-Schindler D, Pfeiffer F, Förstner KU, Hammelmann M, Hilker transcriptional start sites in the haloarchaeon Haloferax volcanii based on differential RNA-Seq (dRNA-Seq). BMC genomics 17:629.

47. Shell SS, Wang J, Lapierre P, Mir M, Chase MR, Pyle MM, Gawande R, Ahmad R, 
593 48. Belnap CP, Pan C, Denef VJ, Samatova NF, Hettich RL, Banfield JF. 2011. Quantitative

594 proteomic analyses of the response of acidophilic microbial communities to different $\mathrm{pH}$

595 conditions. The ISME journal, 2011 ed. 5:1152-1161.

49. Parkhomchuk D, Borodina T, Amstislavskiy V, Banaru M, Hallen L, Krobitsch S, Lehrach

H, Soldatov A. 2009. Transcriptome analysis by strand-specific sequencing of complementary DNA. Nucleic acids research, 2009 ed. 37:e123.

50. Quast C, Pruesse E, Yilmaz P, Gerken J, Schweer T, Yarza P, Peplies J, Glöckner FO. 2013. The SILVA ribosomal RNA gene database project: improved data processing and web-based tools. Nucleic acids research 41:D590-6.

51. Langmead B, Salzberg SL. 2012. Fast gapped-read alignment with Bowtie 2. Nat Methods 9:357-359.

52. Acuna LG, Cardenas JP, Covarrubias, P. C., Haristoy JJ, Flores R, Nunez H, Riadi G, Shmaryahu A, Valdes J, Dopson M, Rawlings DE, Banfield JF, Holmes DS, Quatrini R. 2013. Architecture and gene repertoire of the flexible genome of the extreme acidophile Acidithiobacillus caldus. PloS one, 2013 ed. 8:e78237. 
volcanic ash deposit on the island of Miyake, Japan. Journal of bacteriology, 2012 ed. 194:41224123.

55. Lo I, Denef VJ, Verberkmoes NC, Shah MB, Goltsman D, DiBartolo G, Tyson GW, Allen EE, Ram RJ, Detter JC, Richardson P, Thelen MP, Hettich RL, Banfield JF. 2007. Strainresolved community proteomics reveals recombining genomes of acidophilic bacteria. Nature,

56. Goltsman DSA, Dasari M, Thomas BC, Shah MB, VerBerkmoes NC, Hettich RL, Banfield 2007 ed. 446:537-541.

57. Allen EE, Tyson GW, Whitaker RJ, Detter JC, Richardson PM, Banfield JF. 2007. Genome dynamics in a natural archaeal population. Proceedings of the National Academy of Sciences of the United States of America, 2007 ed. 104:1883-1888.

58. Baker BJ, Comolli LR, Dick GJ, Hauser LJ, Hyatt D, Dill BD, Land ML, Verberkmoes NC, Hettich RL, Banfield JF. 2010. Enigmatic, ultrasmall, uncultivated Archaea. Proceedings of the National Academy of Sciences of the United States of America, 2010 ed. 107:8806-8811.

59. Yelton AP, Comolli LR, Justice NB, Castelle C, Denef VJ, Thomas BC, Banfield JF. 2013. Comparative genomics in acid mine drainage biofilm communities reveals metabolic and structural differentiation of co-occurring archaea. BMC genomics, 2013 ed. 14:485.

60. Andersson AF, Banfield JF. 2008. Virus population dynamics and acquired virus resistance in natural microbial communities. Science 320:1047-1050. annotated genomes using RNA-Seq. Bioinformatics, 2011 ed. 27:2325-2329. 
62. Griffiths-Jones S, Bateman A, Marshall M, Khanna A, Eddy SR. 2003. Rfam: an RNA family database. Nucleic acids research, 2003rd ed. 31:439-441. Artemis: sequence visualization and annotation. Bioinformatics, 2000 ed. 16:944-945.

64. Altschul SF, Gish W, Miller W, Myers EW, Lipman DJ. 1990. Basic local alignment search tool. J Mol Biol, 1990 ed. 215:403-410.

65. Solovyev VV, Salamov A. 2011. Automatic Annotation of Microbial Genomes and Metagenomic Sequences. In Li, RW (ed.), Metagenomics and its applications in agriculture, biomedicine, and environmental studies, 1st ed. Nova Science Publishers, Inc.

66. Lesnik EA, Sampath R, Levene HB, Henderson TJ, McNeil JA, Ecker DJ. 2001. Prediction of rho-independent transcriptional terminators in Escherichia coli. Nucleic acids research

67. Abreu-Goodger C, Merino E. 2005. RibEx: a web server for locating riboswitches and other conserved bacterial regulatory elements. Nucleic acids research 33:W690-2.

68. Hofacker IL. 2003. Vienna RNA secondary structure server. Nucleic acids research 31:34293431.

69. Team RC. R: A language and environment for statistical computing. R Foundation for Statistical Computing. 2017. data. BMC bioinformatics 12:480. RNA-seq data with DESeq2. Genome Biol 15:550. 
661 72. Eisen MB, Spellman PT, Brown PO, Botstein D. 1998. Cluster analysis and display of genome-

662 wide expression patterns. Proceedings of the National Academy of Sciences of the United States

663 of America 95:14863-14868.

664 73. Saldanha AJ. 2004. Java Treeview--extensible visualization of microarray data. Bioinformatics $665 \quad 20: 3246-3248$.

666 74. Dereeper A, Guignon V, Blanc G, Audic S, Buffet S, Chevenet F, Dufayard JF, Guindon S, 667 Lefort V, Lescot M, Claverie JM, Gascuel O. 2008. Phylogeny.fr: robust phylogenetic analysis 668 for the non-specialist. Nucleic acids research, 2008 ed. 36:W465-9.

669 75. Skennerton CT, Imelfort M, Tyson GW. 2013. Crass: identification and reconstruction of 670 CRISPR from unassembled metagenomic data. Nucleic acids research, 2013 ed.

671 76. Goltsman DSA. Richmond Mine Acid Mine Drainage Transcriptome or Gene expression.

672 NCBI GenBank. (accession no. SRP026490).

$673 \quad$ https://trace.ncbi.nlm.nih.gov/Traces/sra/?study=SRP026490 


\section{TABLES}

677 Table 1. Summary statistics and description of samples sequenced. Samples were collected

678 directly from the Richmond Mine (Env) or from bioreactor-grown biofilms (BR). GS: growth

679 stage; Temp: temperature; Type of sequencing: GAII, Illumina GAIIx platform; HiSeq, Illumina

680 HiSeq 2500 platform.

681

\begin{tabular}{|c|c|c|c|c|c|c|c|c|c|c|c|c|}
\hline \multirow[b]{2}{*}{ Sample ID } & \multirow[b]{2}{*}{$\begin{array}{c}\text { Date } \\
\text { collected }\end{array}$} & \multirow[b]{2}{*}{$\begin{array}{l}\text { Env } \\
\text { Type }\end{array}$} & \multirow[b]{2}{*}{ GS } & \multirow[b]{2}{*}{ Location } & \multirow[b]{2}{*}{ Temp } & \multirow[b]{2}{*}{$\mathrm{pH}$} & \multirow[b]{2}{*}{$\begin{array}{l}\text { Type } \\
\text { seq. }\end{array}$} & \multicolumn{2}{|c|}{ Total RNA } & \multicolumn{2}{|c|}{ rRNA-subtracted } & \multirow[b]{2}{*}{$\begin{array}{r}\text { Non- } \\
\text { rRNA } \\
\text { Reads (M) }\end{array}$} \\
\hline & & & & & & & & $\begin{array}{r}\text { No. } \\
\text { reads } \\
(\mathrm{M}) \\
\end{array}$ & $\begin{array}{r}\% \\
\text { rRNA } \\
\end{array}$ & $\begin{array}{r}\text { No. } \\
\text { Reads } \\
(\mathrm{M}) \\
\end{array}$ & $\begin{array}{r}\% \\
\text { rRNA } \\
\end{array}$ & \\
\hline R1_GS0 & $3 / 30 / 10$ & BR & 0 & Outflow & 37 & $\mathrm{NA}^{*}$ & GAII & 2.56 & 90.55 & 4.27 & 84.85 & 0.89 \\
\hline R1_GS05 & $2 / 19 / 10$ & $\mathrm{BR}$ & 0.5 & Outflow & 37 & 1.65 & GAII & 4.86 & 88.34 & - & - & 0.57 \\
\hline R2_GS05 & $7 / 20 / 09$ & BR & 0.5 & A drift & 37 & $\mathrm{NA}^{*}$ & GAII & 4.79 & 84.97 & - & - & 0.72 \\
\hline A-drift & 9/17/10 & Env & 0 & A drift & 40 & 1.27 & GAII & 3.41 & 86.00 & - & - & 0.48 \\
\hline C75_GS1 & $9 / 17 / 10$ & Env & 1 & C drift & 46 & 0.86 & GAII & 4.66 & 90.58 & 4.59 & 77.17 & 1.49 \\
\hline AB10_GS0 & $11 / 2 / 10$ & Env & 0 & $\mathrm{AB}$ drift & 39 & 0.8 & HiSeq & 27.87 & 96.22 & 53.15 & 82.09 & 10.57 \\
\hline AB10_GS1 & $11 / 2 / 10$ & Env & 1 & $\mathrm{AB}$ drift & 39 & 0.8 & HiSeq & 30.24 & 96.46 & 58.42 & 81.82 & 11.69 \\
\hline C10_GS0 & $11 / 2 / 10$ & Env & 0.5 & $\mathrm{C}$ drift & 42 & 0.8 & HiSeq & 30.91 & 94.12 & - & - & 1.60 \\
\hline C10_GS05 & $11 / 2 / 10$ & Env & 0 & $\mathrm{C}$ drift & 42 & 0.8 & HiSeq & 27.12 & 96.16 & 51.88 & 85.39 & 8.77 \\
\hline C10_GS1 & $11 / 2 / 10$ & Env & 1 & $\mathrm{C}$ drift & 42 & 0.8 & HiSeq & 28.60 & 95.49 & 50.65 & 85.66 & 8.55 \\
\hline G2E1_GS2 & $6 / 9 / 09$ & Env & 2 & 4-way & 39 & 0.7 & HiSeq & 26.68 & 85.73 & 45.06 & 68.76 & 17.88 \\
\hline R3_GS0 & $9 / 28 / 10$ & BR & 0 & A drift & 37 & 1.31 & HiSeq & 31.56 & 95.00 & - & - & 1.58 \\
\hline R3_GS1 & $10 / 6 / 10$ & BR & 1 & A drift & 37 & 1.74 & HiSeq & 30.49 & 94.08 & 67.98 & 75.10 & 18.73 \\
\hline
\end{tabular}

$682 *$ NA indicates that no $\mathrm{pH}$ measurements were available for these samples. 


\section{FIGURE LEGENDS}

686 Figure 1. (next page) Differential gene and genome expression correlates with environmental 687 conditions in AMD biofilms. A) Relative expression abundance of AMD genomes. Read counts 688 were normalized by the genome length and sample size. B) Proportion of AMD genomes covered 689 by transcriptomic reads. The top 20 most abundant taxa and viruses are shown.

691 Figure 2. Non-metric multidimensional scaling (NMDS) ordination on Bray-Curtis distances of 692 differentially expressed genes in AMD communities. A) NMDS samples plot color coded by $\mathrm{pH}$ 693 and environment type. B-D) NMDS genes plot colored by: B) the top most abundant 694 Leptospirillum species and strains; C) the top most abundant Archaeal species, and D) viral 695 genomes. NMDS genes plots indicate a gradient of points going from the lower right quadrant to 696 the top right quadrant, which then goes to the top left quadrant (horseshoe).

698 Figure 3. (previous page) Environmental conditions distinguish expression of groups of genes 699 and functional categories in AMD systems. A-D) Heatmaps of genes with log2-fold change $>5$ 700 in: A) Leptospirillum group II UBA; B) G-plasma; C) Leptospirillum group III; and D)

701 Leptospirillum group II 5way CG. Yellow: overrepresented, blue: underrepresented. E)

702 Functional categories differ in communities growing in the natural environment vs. laboratory

703 bioreactors. Categories enriched in environmental samples are shown in dark blue, while

704 categories enriched in bioreactor samples are shown in orange. Functional categories in bold 705 within black boxes represent the categories in which the most variation between conditions was 706 observed. 
708 Figure 4. Predicted secondary structures of transcribed ncRNAs of known function in

709 Leptospirillum sp. A) Sequence logo representation of the promoter structure in Leptospirillum

710 group II UBA genotype. The alignment was constructed using the sequences of 207 predicted

711 promoters. B-C) Secondary structures predicted in Leptospirillum group II UBA (B, top

712 structures) and in Leptospirillum group III (C, bottom structures). Secondary structure prediction

713 was done on the RNAfold webserver.

715 Figure 5. Expression of regulatory ncRNAs in Leptospirillum group II. A) Modified Artemis 716 image showing the transcriptional expression of Leptospirillum group II UBA CRISPR loci.

717 Expression of Cas genes occurs in the same orientation as the predicted genes on scaffold 8241

718 (orange line, (-) strand), while pre-crRNAs are cis-transcribed on the antisense strand (green line,

719 (+) strand). CPUF: conserved hypothetical protein; TR: transcriptional regulator; TN:

720 transposase. Pre-crRNAs are shown as blue boxes, predicted coding genes are represented by

721 teal boxes, the CRISPR leader sequence (L) is represented by a black box downstream of Cas2

722 and is followed by the first repeat in the composite assembly (red box). The last repeat of the loci

723 is also show as a red box next to the transcriptional regulator. The composite assembled CRISPR

724 locus, including the Cas genes, is $\sim 25 \mathrm{~Kb}$ in length. B-C) Novel ectoine riboswitch in

725 Leptospirillum group II bacteria. The ectoine operon (teal blue arrows), and the riboswitch

726 (ncRNA, white box) with its promoter $(\mathrm{P})$ and Rho-independent terminator $(\mathrm{T})$ are shown. The

727 modified Artemis image in Leptospirillum group II UBA (B, top) and in Leptospirillum group II

728 5way -CG (B, bottom) shows the strand-specific transcriptional expression from two bioreactor

729 samples (blue: R3_GS0; magenta: R3_GS1) and two environmental biofilms (red: AB10_GS0;

730 green: AB10_GS1). The predicted secondary structures of the riboswitches are shown in: 
731 Leptospirillum group II UBA (C, top) and in Leptospirillum group II 5way CG (C, bottom) with

732 their Rho-independent terminator (solid arrow) and an anti-terminator (dashed arrow).

734 Figure 6. Expression of regulatory ncRNAs in Leptospirillum group III. Panels show the strand-

735 specific expression of energy generation-related genes with their associated regulatory ncRNA.

736 Coding genes are represented by teal arrows, ncRNAs by white arrows, putative uORFs by blue

737 arrows, and promoters by green arrows. Left: CO dehydrogenase (CODH) beta subunit with its

738 transcribed short (70 bp) ncRNA. The predicted secondary structure for the CODH-ncRNA is

739 shown. Purple and green lines: R3_GS1 bioreactor biofilm; blue and teal lines: R3_GS0

740 bioreactor biofilm. Middle: pyruvate:ferredoxin oxidorectase (PFOR) operon with its transcribed

7415 ' UTR containing a putative promoter (P, green) and possible regulatory uORFs. Right:

742 Cytochrome c 572 (Cyt572) with its transcribed 5' UTR containing a putative promoter (P) and

743 possible regulatory uORFs. The length of the putative regulatory uORFs ranges from 10 to 40

744 amino acids long. 


\section{SUPPLEMENTARY MATERIALS LEGENDS}

748 1. Supplementary Table S1. Genome-wide relative abundance of transcripts from AMD

749 genomes.

750 2. Supplementary Table S2. Predicted promoters upstream of transcribed genes in $751 \quad$ Leptospirillum group II UBA genotype.

752 3. Supplementary Table S3. Transcripts containing ncRNAs with predicted functions.

753 4. Supplementary Table S4. Summary of CRISPR spacers recovered from paired-end, strand$754 \quad$ specific transcriptomics datasets.

755 5. Supplementary Table S5. CRISPR CRASS output table and spacer matches to previous 756 CRISPR analyses.

757 6. Supplementary Figure S1. Differential expression analysis of genes from AMD genomes.

758 7. Supplementary Figure S2. Gene tree of transposases expressed in Leptospirillum sp.

759 8. Supplementary Figure S3. Examples of expression of leadered transcripts in Leptospirillum 760 sp.

761 9. Supplementary Figure S4. Transcript abundance plots from total vs. rRNA-depleted RNA 762 samples.

763 
bioRxiv preprint doi: https://doi.org/10.1101/538918; this version posted February 2, 2019. The copyright holder for this preprint (which was not certified by peer review) is the author/funder, who has granted bioRxiv a license to display the preprint in perpetuity. It is made available under aCC-BY-ND 4.0 International license.

\section{FIGURES}

A.

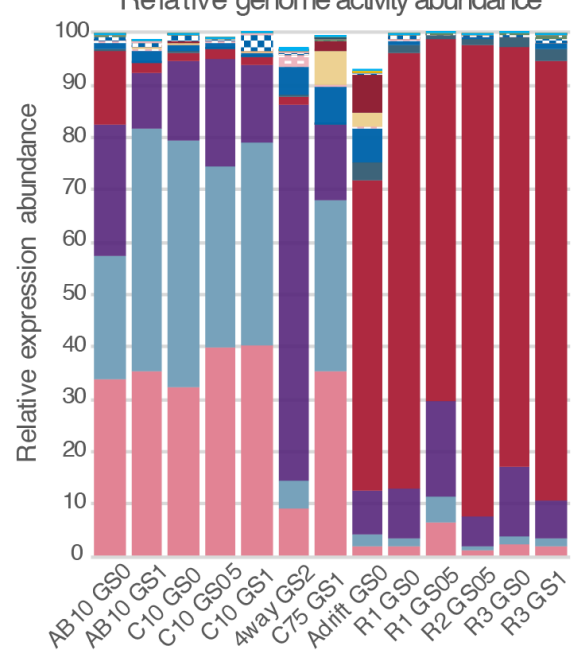

B. Proportion of the genome with transcripts
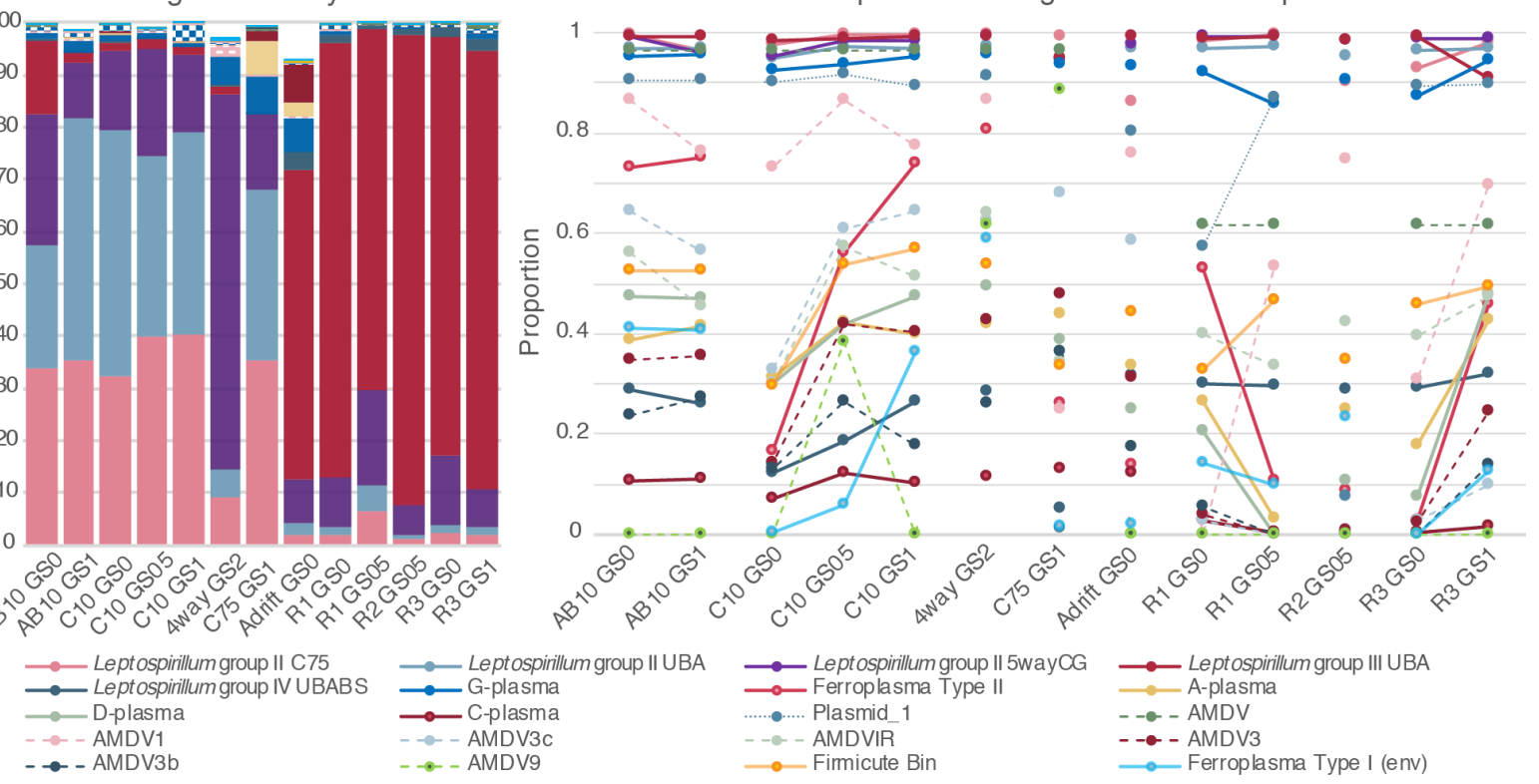

767 Figure 1. Transcriptional expression of acid mine drainage genomes. A) Relative genome activity of

768 AMD genomes across samples. Read counts were normalized by the genome length and sample size.

769 B) Proportion of AMD genomes covered by transcriptomic reads. The top 20 most abundant taxa

770 and viruses are shown on both panels. 
A. Samples

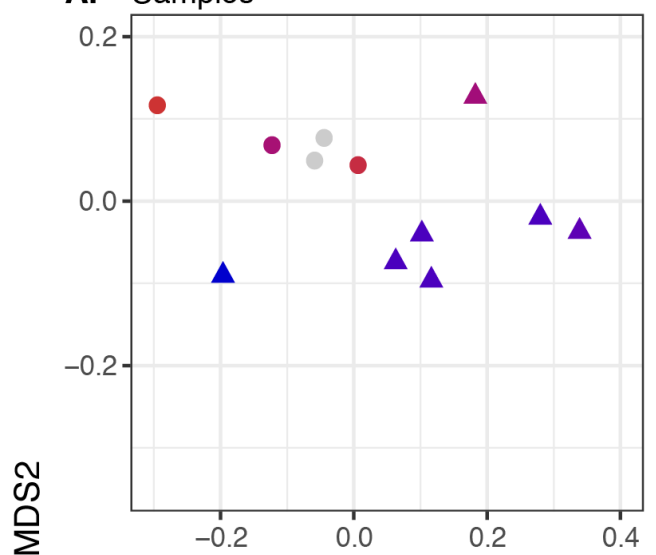

C. Archaea

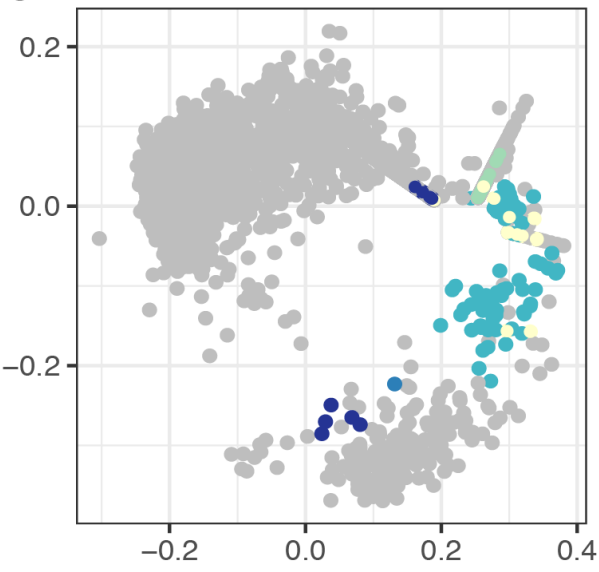

772

773 Figure 2. Non-metric multidimensional scaling (NMDS) ordination on Bray-Curtis distances of

774 differentially expressed genes in AMD communities. A) NMDS samples plot coded by $\mathrm{pH}$ and

775 environment type. B-D) NMDS genes plot colored by: B) Leptospirillum species and strains; C)

776 Archaeal species, and D) viral genomes. NMDS genes plots indicate a gradient of points going from

777 the lower right quadrant towards the top left quadrant (horseshoe).
B. Leptospirillum

D. Viruses

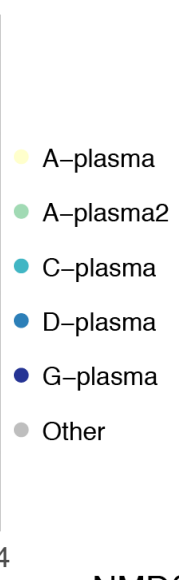

NMDS1

- BR

- Env

$\mathrm{pH}$

$-1.25$ 0.75

$-0.2$
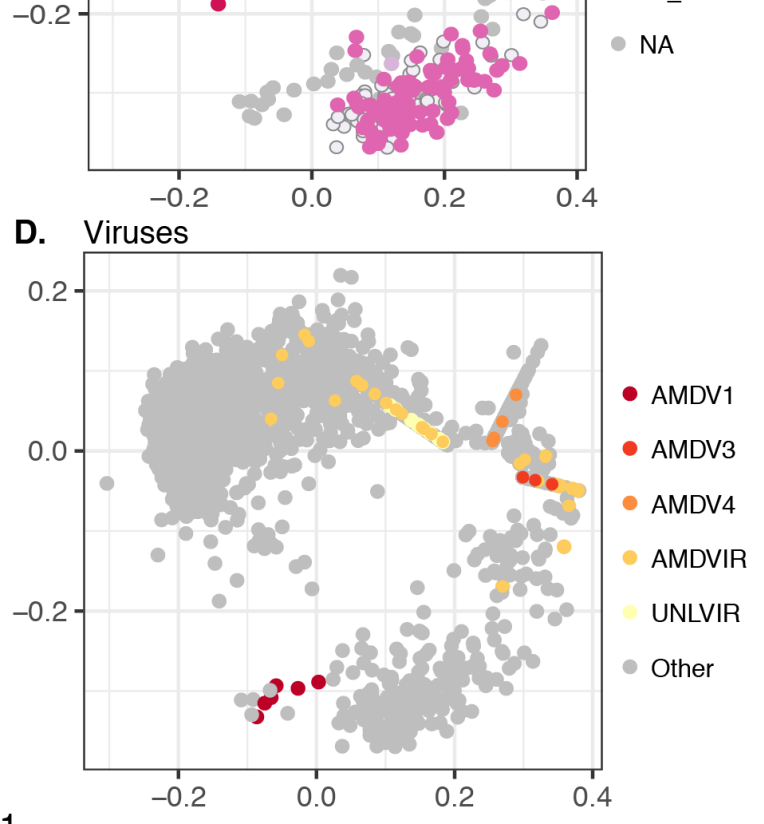

L3_UBA

NA

L2_CG

L2_UBA

AMDV3

AMDVIR UNLVIR 
bioRxiv preprint doi: https://doi.org/10.1101/538918; this version posted February 2, 2019. The copyright holder for this preprint (which was not certified by peer review) is the author/funder, who has granted bioRxiv a license to display the preprint in perpetuity. It is made available under aCC-BY-ND 4.0 International license.

A.

E.

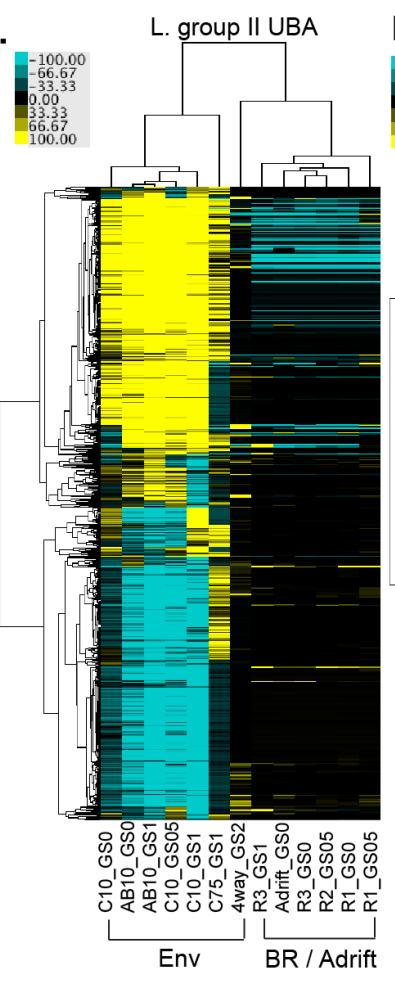

B.

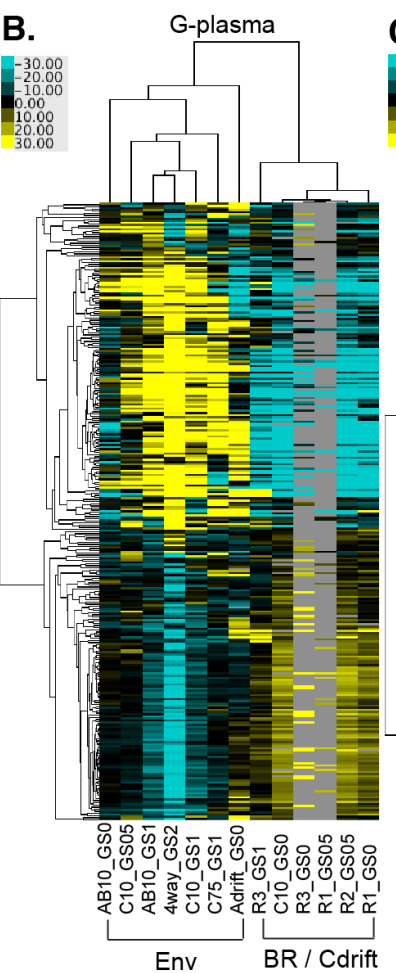

C.

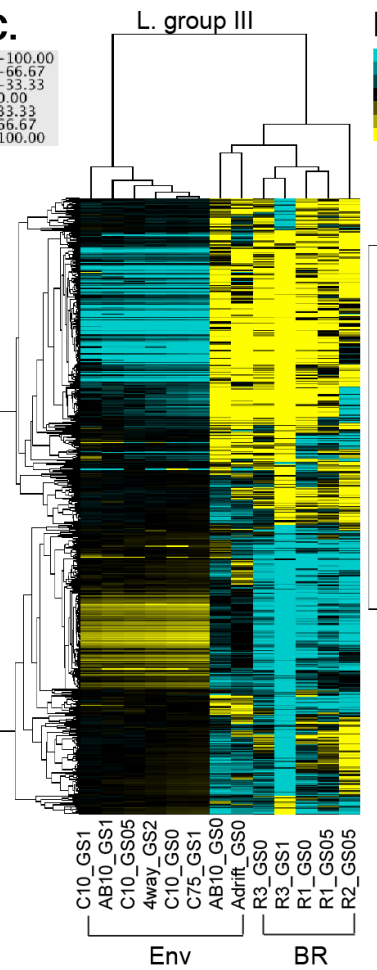

D. L. group II 5way CG

L. group II UBA

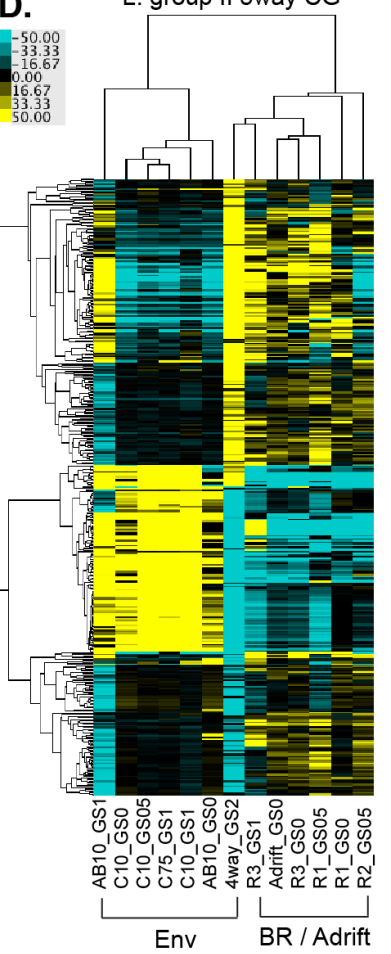

Amino acid and cofactor metabolism

Carbohydrate and lipid metabolism Cell division "." Chaperones and folding Chemotaxis CRISPR

DNA repair and recombination Energy production and conversion Enzymatic reactions Fatty acid metabolism Flagella Hydrolases Unknown Function Lipopolysaccharide metabolism Secondary metabolites pathways Nucleotide metabolism Peptidoglycan biosynthesis Phosphate metabolism

Regulation Replication Ribosomal proteins Ribosome biogenesis RNA processing Signal Transduction Stress and Resistance Sulfur Metabolism

Mobile elements

Transcription factors Transcription machinery Transferases Translation Factors Transport tRNA biogenesis and modification "w"

tRNA Synthetases "i"

Vitamin Metabolism Xenobiotics Metabolism

G-plasma

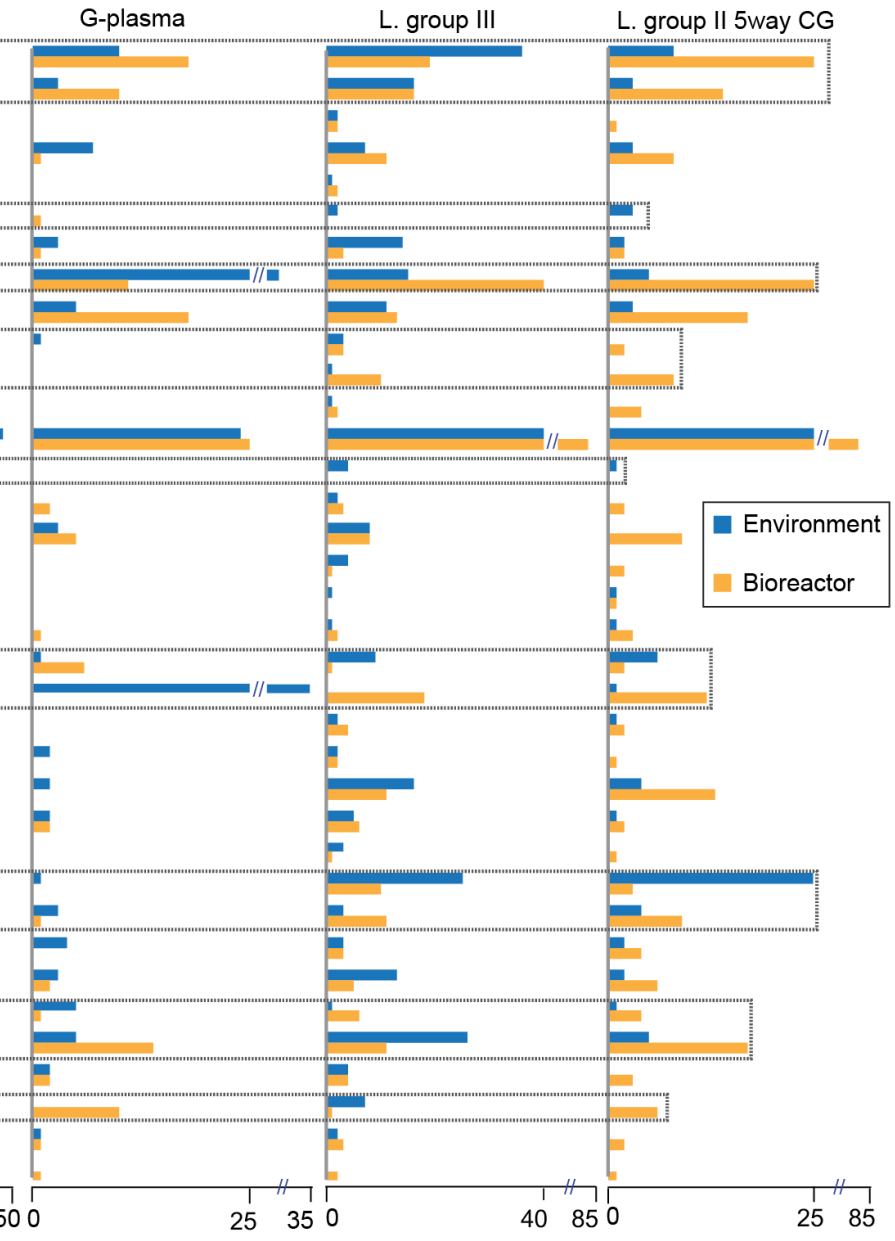


779 Figure 3. (previous page) Environmental conditions distinguish expression of groups of genes and

780 functional categories in AMD systems. A-D) Heatmaps of genes with log2-fold change > 5 in: A)

781 Leptospirillum group II UBA; B) G-plasma; C) Leptospirillum group III; and D) Leptospirillum group

782 II 5way-CG. Yellow: overrepresented, blue: underrepresented. E) Functional categories differ in

783 communities growing in the natural environment vs. laboratory bioreactors. Categories enriched

784 in environmental samples are shown in dark blue, while categories enriched in bioreactor samples

785 are shown in orange. Functional categories in bold within black boxes represent the categories in 786 which the most variation between conditions was observed. 
A.
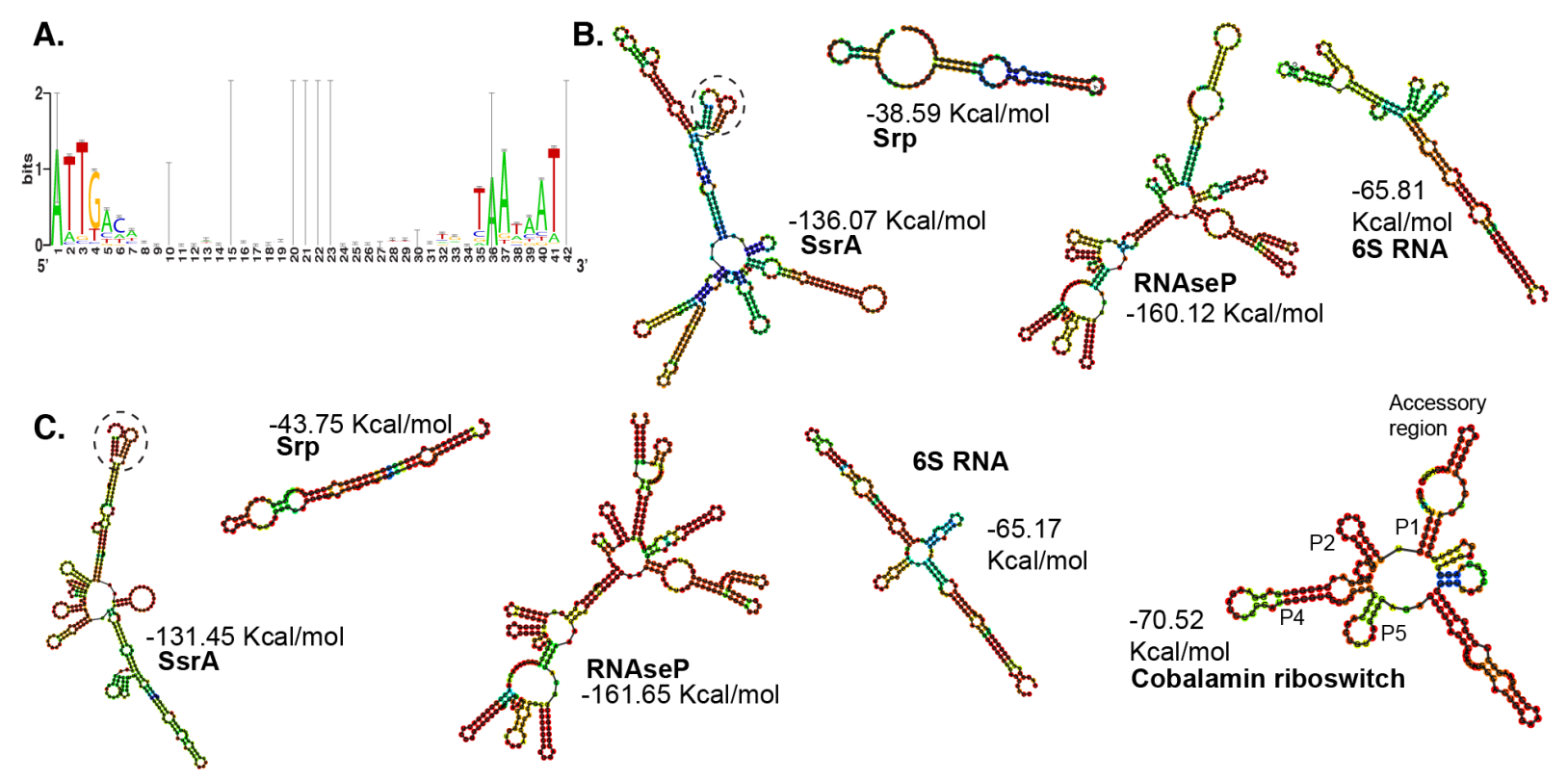

788 Figure 4. Predicted secondary structures of transcribed ncRNAs of known function in Leptospirillum

789 sp. A) Sequence logo representation of the promoter structure in Leptospirillum group II UBA genotype.

790 The alignment was constructed using the sequences of 207 predicted promoters. B) structures

791 predicted in Leptospirillum group II UBA (Top structures). C) Structures predicted in Leptospirillum

792 group III. Secondary structure prediction was done on the RNAfold webserver.

793

794 

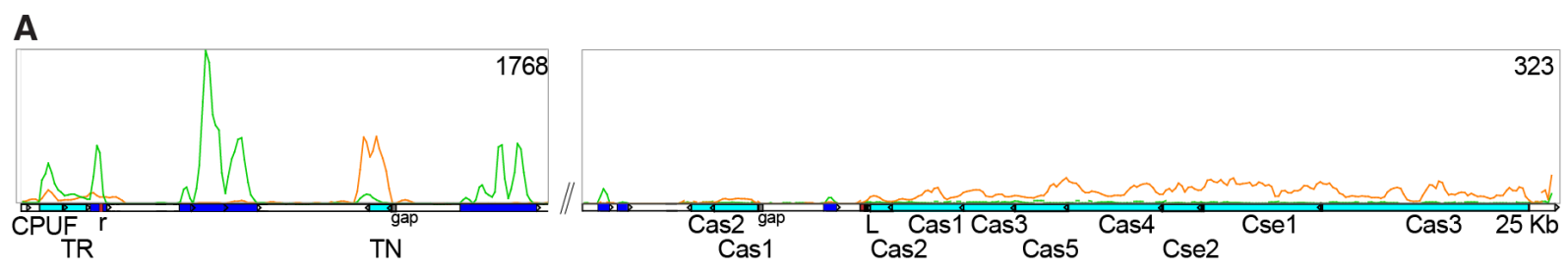

B
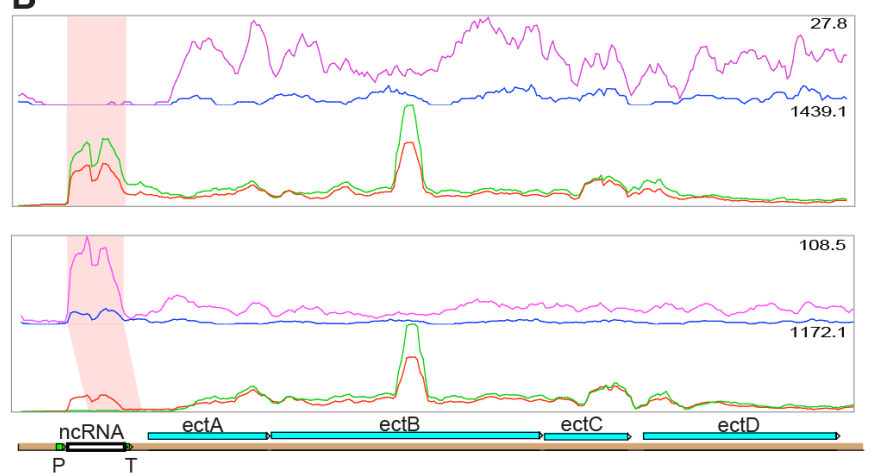

C

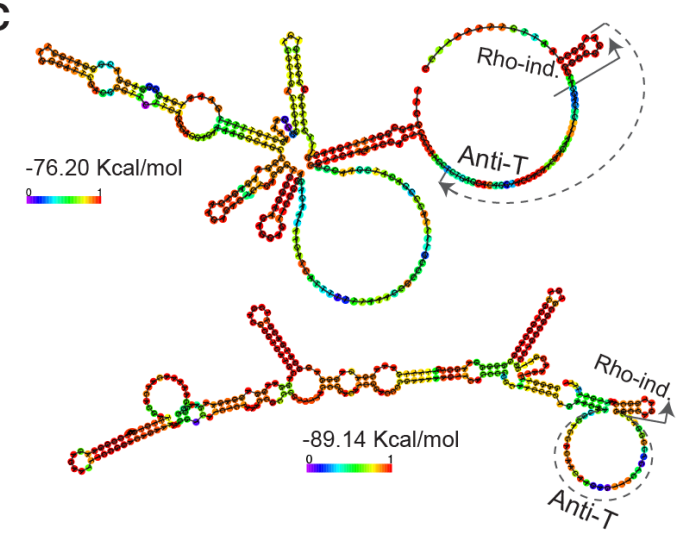

Figure 5. Expression of regulatory ncRNAs in Leptospirillum group II. A) Modified Artemis image showing the transcriptional expression of Leptospirillum group II UBA CRISPR loci. Expression of Cas genes occurs in the same orientation as the predicted genes on scaffold 8241 (orange line, (-) strand), while pre-crRNAs are cis-transcribed on the antisense strand (green line, (+) strand). CPUF: conserved

800 hypothetical protein; TR: transcriptional regulator; TN: transposase. Pre-crRNAs are shown as blue 801 boxes, predicted coding genes are represented by teal boxes, the CRISPR leader sequence (L) is

802 represented by a black box downstream of Cas2 and is followed by the first repeat in the composite 803 assembly (red box). The last repeat of the loci is also show as a red box next to the transcriptional 804 regulator. The composite assembled CRISPR locus, including the Cas genes, is $\sim 25 \mathrm{~Kb}$ in length. B-C)

805 Novel ectoine riboswitch in Leptospirillum group II bacteria. The ectoine operon (teal blue arrows), and 806 the riboswitch (ncRNA, white box) with its promoter (P) and Rho-independent terminator (T) are 807 shown. The modified Artemis image in Leptospirillum group II UBA (B, top) and in Leptospirillum group 808 II 5way -CG (B, bottom) shows the strand-specific transcriptional expression from two bioreactor 809 samples (blue: R3_GS0; magenta: R3_GS1) and two environmental biofilms (red: AB10_GS0; green: 810 AB10_GS1). The predicted secondary structure of the riboswitches are shown in: Leptospirillum group 
811 II UBA (C, top) and in Leptospirillum group II 5way CG (C, bottom) with their Rho-independent

812 terminator (solid arrow) and an anti-terminator (dashed arrow).
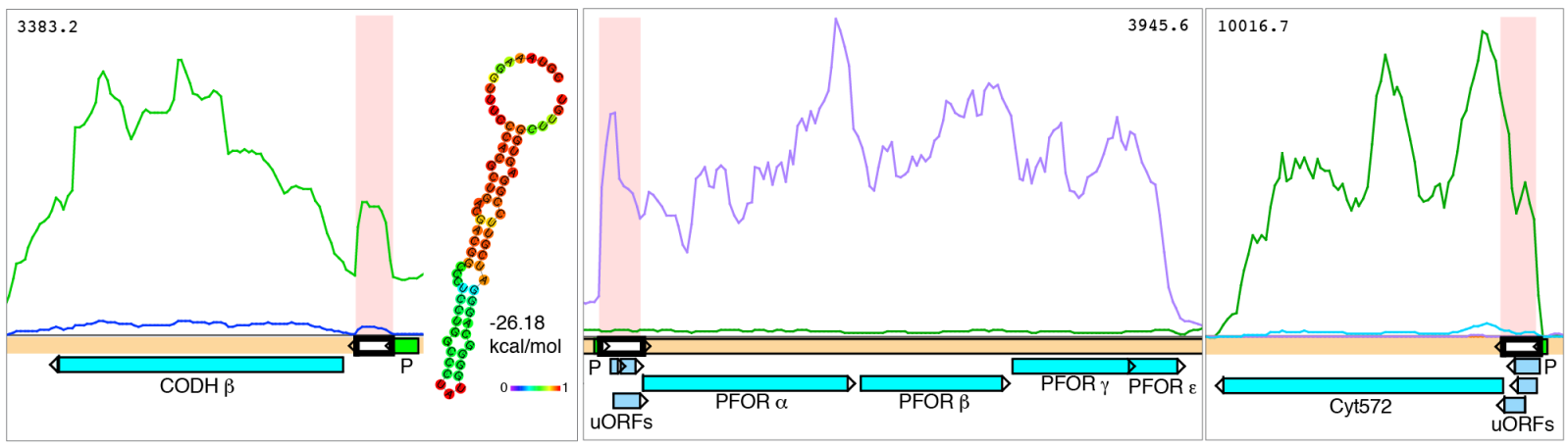

814 Figure 6. Expression of regulatory ncRNAs in Leptospirillum group III. Panels show the strand-specific

815 expression of energy generation-related genes with their associated regulatory ncRNA. Coding genes

816 are represented by teal arrows, ncRNAs by white arrows, putative uORFs by blue arrows, and

817 promoters by green arrows. Left: $\mathrm{CO}$ dehydrogenase (CODH) beta subunit with its transcribed short

818 (70 bp) ncRNA. The predicted secondary structure for the CODH-ncRNA is shown. Purple and green

819 lines: R3_GS1 bioreactor biofilm; blue and teal lines: R3_GS0 bioreactor biofilm. Middle:

820 pyruvate:ferredoxin oxidorectase (PFOR) operon with its transcribed 5' UTR containing a putative

821 promoter (P, green) and possible regulatory uORFs. Right: Cytochrome c 572 (Cyt572) with its

822 transcribed 5' UTR containing a putative promoter (P) and possible regulatory uORFs. The length of

823 the putative regulatory uORFs ranges from 10 to 40 amino acids long.

824

825 